\title{
Wer hat, dem wird gegeben? Zur Problematik von Matthäuseffekten in Förderprogrammen
}

\section{Margrit Stamm}

Förderprogramme haben häufig den überraschenden Effekt, dass sie Unterschiede zwischen leistungsstarken und leistungsschwachen Schülern nicht wie beabsichtigt verkleinern, sondern eher vergrössern ("Matthäuseffekte»). Dieser Aufsatz diskutiert zunächst empirisches Belegmaterial für dieses Phänomen unter besonderer Berücksichtigung der unterschiedlichen Lernzuwächse in Abhängigkeit der besuchten Schulform. Sodann stellt er auf der Basis der Befunde zweier Evaluationsstudien ein Taxonomiemodell für disproportionale Gewinne in Förderprogrammen vor, das sich auf leistungsbasiert-individuelle und auf zugangsbasiert-strukturelle Effekte abstützt. Anschliessend werden einige differenzialpsychologischen und sozialpolitischökonomischen Fragen diskutiert, wie grundsätzlich mit Matthäuseffekten umgegangen werden soll, wenn kompensatorische Massnahmen nicht die intendierten Effekte erzielen. Abschliessend werden einige pädagogischen Konsequenzen formuliert, welche einen Beitrag zu ihrer Egalisierung leisten könnten.

Unsere deutschsprachigen Länder geben jedes Jahr Millionen aus, um die Ausbildungsdefizite auszubessern und Ausbildungschancen zu optimieren. Im Mittelpunkt stehen dabei Projekte zur Sprachförderung, Strategieprogramme für selbstgesteuertes Lernen, integrierte Begabungsförderangebote oder Interventionsprogramme für Lehrstellensuchende. Viele dieser Programme haben zum Ziel, die Unterschiede zwischen leistungsstarken und leistungsschwachen Schülerinnen und Schülern auszugleichen. Die Grundlage dieser Unterschiede ist der vielfach beschriebene Matthäuseffekt, eine neutestamentliche Parabel bei Matthäus XXV, wo es im Vers 29 heisst: «Denn, wer hat, dem wird gegeben, und er wird im Überfluss haben, wer aber nicht hat, dem wird noch weggenommen, was er hat.» Gemeint ist damit das vielfach zu beobachtende Phänomen, dass ein initiales Privileg (beispielsweise ökonomisches Kapital oder kognitive Fähigkeiten) zu kumulativen Gewinnen führt und bereits bestehende Unterschiede durch die Privilegierung von Chancenstrukturen verstärkt. Weinert (2000) hat dieses Phänomen für den schulischen Leistungsbereich umschrieben: «Wer zu einem bestimmten Zeitpunkt im Vergleich zu anderen über bessere individuelle 
Lernvoraussetzungen verfügt, wird von gleichen Lernangeboten und Lerngelegenheiten stärker profitieren. Dies führt notwendigerweise zu Schereneffekten in der kognitiven Entwicklung, d. h. zu einer Spreizung der individuellen Leistungsunterschiede» (S. 16). Matthäuseffekte sind bislang in vielen Bereichen beobachtet worden, so zunächst in der Akkumulation wissenschaftlichen Prestiges von Merton (1968), in den letzten Jahren jedoch auch in der Weiterbildung (Barz \& Tippelt, 2004; Gruber, 2001) in der Evaluationsforschung (Bessoth, 1994), vor allem jedoch auch in der Forschung zu Lernverläufen in unterschiedlichen Schulformen (Becker, Lüdtke, Trautwein \& Baumert, 2006). Zwar liegen inzwischen auch einige Versuche vor, solche Schereneffekte zu erklären (Baumert, Stanat \& Watermann, 2006). Im Gegensatz zum angloamerikanischen Sprachraum (Deci \& Papierno, 2005; Stanovich, 1986; Walberg \& Tsai, 1983) stehen jedoch hierzulande kaum Analysen zur Verfügung, welche Matthäuseffekte vertieft und systematisch analysieren und auch der Frage nachgehen, wie sie abgebaut werden könnten.

Hier setzt dieser Aufsatz ein. Im ersten Kapitel diskutiert er empirisches Belegmaterial für Matthäuseffekte unter besonderer Berücksichtigung der unterschiedlichen Lernzuwachse in Abhängigkeit der besuchten Schulform. Kapitel 2 stellt eine Taxonomie für disproportionale Gewinne in Förderprogrammen vor, die sich auf leistungsbasiert-individuelle und auf zugangsbasiert-strukturelle Effekte abstützt. Auf dieser Basis werden in Kapitel 3 einige differenzialpsychologische und sozialpolitisch-ökonomische Fragen diskutiert, wie mit Matthäuseffekten grundsätzlich umgegangen werden soll, wenn kompensatorische Massnahmen nicht die intendierten Effekte erzielen. Die Beantwortung dieser Fragen führt in Kapitel 4 zur Formulierung einiger pädagogischer Konsequenzen, welche einen Beitrag zu ihrer Egalisierung leisten könnten.

\section{Empirische Belege für oder gegen Matthäuseffekte}

Das Matthäus-Phänomen ist nicht neu und für die Erziehungswissenschaften bereits vor mehr als zwanzig Jahren von Stanovich (1986, S. 380ff.) als «organism-environment-correlation» in Anlehnung an die Typologie der Anlage-Umwelt-Korrelation von Plomin, DeFries und Loehlin (1977) beschrieben worden. Er wollte damit verdeutlichen, dass Disparitäten zunehmen, wenn Kinder mit unterschiedlichen Genotypen selektiv unterschiedlichen Umgebungstypen ausgesetzt sind.

Neu ist allerdings, dass verschiedene Untersuchungen in der Zwischenzeit aufzeigen konnten, wie frühere Vorteile über die Zeit hinweg akkumuliert werden. Nachdem erste Untersuchungen zum schulischen Leistungsausgleich von Treiber und Weinert (1985) oder zu Unterrichtsqualität und Leistungszuwachs von Helmke und Weinert (1987) Hinweise auf Matthäuseffekte geliefert hatten, können wir spätestens seit den Scholastik- und Logik-Untersuchungen von Wei- 
nert (1997) sowie Weinert und Helmke (1997) nicht nur die Frage «Wie durchlaufen Schülerinnen und Schüler ihren schulischen Bildungsweg?» klarer beantworten, sondern auch belegen, dass diejenigen, welche bereits privilegiert sind, durch die Multiplikation ihres intellektuellen, ökonomischen und sozialen Kapitals noch privilegierter zu werden scheinen und sich die Unterschiede über die Zeit hinweg vergrössern. In allen diesen Studien belegen die Leistungsentwicklungen die Stabilität interindividueller Unterschiede über die gesamte Schullaufbahn hinweg. Auch unter dem Einfluss extern angeregter und geförderter Lernprozesse bleiben die Differenzen bestehen. Schereneffekte liessen sich auch in der internationalen Vergleichsstudie von Willms (2006) nachweisen, der sozioökonomische Gradienten auf der Basis von PIRLS- und PISA-Ergebnissen in den teilnehmenden Ländern untersuchte. Dabei zeigte sich für weniger erfolgreiche Länder, dass Kinder aus sozioökonomisch gut gestellten Familien besonders positive Lernerfahrungen machen, nicht jedoch solche aus eher benachteiligten Milieus und dass erstere darüber hinaus von umfassenderen ausserschulischen Lernmöglichkeiten dank der elterlichen Investitionsfähigkeit profitieren. Schereneffekte sind in der Zwischenzeit von verschiedenen Studien der Arbeitsgruppe um Baumert (Baumert et al., 2006; Becker et al., 2006) empirisch bestätigt worden. Baumert et al. (2006) versuchen, sie anhand dreier Erklärungsansätze zu fundieren: (1) Schülerinnen und Schüler unterschiedlicher Schulformen haben sich bereits vor dem Übertritt in die Sekundarstufe I in ihren Leistungen und Lernraten unterschieden. Die unterschiedlichen Leistungsentwicklungsverläufe wären somit Ausdruck bereits früher angelegter unterschiedlicher Lernkurven (individueller Matthäuseffekt). (2) Es gibt spezifische Fördereffekte der unterschiedlichen Schulformen, die ihre Ursache in unterschiedlichen Stundentafeln, Lehrplänen oder Unterrichtskulturen haben. (3) Es gibt Kompositionseffekte, welche auf die unterschiedliche leistungsmässige, biografische, soziale und kulturelle Zusammensetzung der Schülerschaften zurükkzuführen sind. Somit wären Unterschiede in der Leistungsentwicklung nicht oder nicht allein in der Zugehörigkeit zu einer Schulform, sondern zumindest teilweise in den Charakteristika der jeweiligen Lerngruppe begründet. Ähnliche Befunde liefert die Längsschnittstudie von Stamm (2005). Sie weist nach, dass vorschulische Vorteile in erster Linie bei autodidaktischer Aneignung von Leseund Mathematikvorläuferfertigkeiten zu kumulativen Vorteilen in der nachfolgenden Schullaufbahn führen. Damit illustriert sie ein grundlegendes Prinzip, das auch im Zusammenhang mit dem Experten-Novizen-Paradigma diskutiert worden ist: die Bedeutung des Vorwissens als lernerseitige Voraussetzung für ein angemessenes Leseverständnis und den Erwerb weiterer, darauf aufbauender Information (Chi, Glaser \& Rees, 1982; Voss \& Silfies, 1996). Diese bereits existierende Wissensbasis erlaubt der Person, grössere Expertise mit geringerem Zeitaufwand zu erreichen. Kognitionspsychologie und Unterrichtsforschung weisen nach, dass die bereichsspezifischen Vorkenntnisse die wichtigste Grösse für den schulischen Lernerfolg darstellen, währenddem die moderierenden Ef- 
fekten der Intelligenz eine deutlich geringere Gültigkeit haben. Gemäss Köller und Baumert (2002) beträgt ihr Zusammenhang $r=0.7$, für die Intelligenz jedoch nur $\mathrm{r}=0.3$.

Gegenteilige Befunde liefert die Gruppe um Schneider und Mitarbeitende (Schneider \& Stefanek, 2004). Anhand der Daten aus der LOGIK-Studie verfolgten sie die Leistungsentwicklung von Kindern zwischen dem vierten und dem 17. Lebensjahr. Während sie für die frühe Entwicklungsphase differentielle Verläufe beobachten konnten - Kinder mit bereits zu Beginn der Studie hohen kognitiven Leistungen wiesen auch eine steilere Lernkurve mit zehn Jahren auf blieben die Unterschiede in der zweiten Phase konstant. Schereneffekte blieben auch in einigen Studien der beruflichen Begabungsforschung aus. So prüften Schmidt, Hunter und Outerbridge (1986) anhand dreier Thesen - Divergenzhypothese, Konvergenzhypothese, Noninteraktionshypothese - die Annahme, ob sich höhere Begabung (definiert als allgemeine kognitive Fähigkeiten) zu Beginn der Berufsausbildung in besseren beruflichen Leistungen niederschlägt. Die Divergenzhypothese behauptet eine Zunahme der Leistungsunterschiede mit zunehmender Berufserfahrung, während die Konvergenzhypothese von einer Nivellierung des Berufserfolges zwischen unterschiedlich begabten Personen und die Noninteraktionshypothese von einem konstant bleibenden Vorsprung der Begabteren während der Berufsausbildung ausgeht. Im Ergebnis liessen sich sowohl Belege für die Konvergenz- als auch für die Noninteraktionshypothese finden. Während diese Befunde von weiteren Studien bestätigt werden konnten (Baird, 1985; Hulin, Henry \& Noon, 1992), liessen sich in kaum einer Studie Nachweise für die Divergenzhypothese finden. Dies trifft auch für eine kürzlich abgeschlossene Schweizer Längsschnittstudie zur Entwicklung von Leistungsexzellenz in der beruflichen Ausbildung zu (Stamm, Niederhauser \& Müller, 2009).

Im angelsächsischen Raum existieren zwar viele Studien, welche das Phänomen des Matthäuseffektes spezifisch untersuchten, jedoch beziehen sie sich lediglich auf querschnittliche Daten (Luyten, Cremers-van Wees \& Bosker, 2003). Als grundlegend gelten die Studien von Walberg und Tsai (1983) sowie von Stanovich (1986). Während Walberg und Tsai (1983) das Verdienst zukommt, den Matthäuseffekt erstmals im erziehungswissenschaftlichen Feld bekannt gemacht und ihn mit Bildungsbenachteiligung gleichgesetzt zu haben, gilt dies für Stanovich (1986) in Bezug auf langfristige Matthäus-Effekte in der Leseentwicklung.

Ebenfalls längsschnittig angelegt waren die Studien von Bast und Reitsma (1998), Shaywitz et al. (1995) sowie Aarnoutse und van Leeuwe (2000). Bast und Reitsma (1995) untersuchten in den Niederlanden auf der Basis der Studie von Stanovich (1986) die Lesegewinne von 235 Primarschülern zwischen der zweiten und dritten Klasse. Dabei konnten keine Schereneffekte im Lesen, im Wortschatz und in den Leseinstellungen festgestellt werden, wohl jedoch im Dechiffrieren. Die Studie von Shaywitz et al. (1995) untersuchte 396 Kinder aus Vorschulklassen bis zum Ende des sechsten Schuljahres. Im Ergebnis zeigten sich 
Matthäuseffekte, die sich sowohl auf die Entwicklung des IQs, insbesondere jedoch auch auf das Vorwissen, zurückführen liessen. Aarnoutse und van Leeuwe (2000) verfolgten die Leistungsentwicklungen von 556 Kindern zwischen der dritten und der sechsten Klasse im Leseverständnis, dem Wortschatz und der Aussprache. Sie konnten insgesamt keine Schereneffekte eruieren, weil schwächere Leser eine deutlich ausgeprägter ansteigende Lernkurve als starke Leser verzeichneten. $\mathrm{Zu}$ diesen vier Studien ist allerdings zu bemerken, dass sie weder die sozioökonomische Herkunft berücksichtigten, noch die Entwicklung benachteiligter Kinder in den Blick nahmen. Dieser Frage nahm sich die Metaanalyse von Luyten et al. (2003) an. Auf der Datenbasis eines grossen Samples ( $\mathrm{n}=$ 65'129 niederländische Primarschüler aus 1'555 Schulen) untersuchten sie das Ausmass von Matthäuseffekten zwischen benachteiligt und eher privilegiert aufwachsenden Schülern. Im Ergebnis verwiesen die sprachlichen und mathematischen Lernkurven auf deutliche Schereneffekte zu Gunsten der zweiten Gruppe. Diese Effekte liessen sich auf den Kompetenzstand bei Schuleintritt zurückführen. Interessanterweise war dies bei zwei spezifischen Gruppen - bei türkischen und marokkanischen Schülerinnen und Schülern - nicht der Fall, konnten sie doch vor allem in Mathematik Leistungsgewinne verbuchen.

Insgesamt erweist sich die empirische Befundlage für respektive gegen Matthäuseffekte als widersprüchlich. Diese Widersprüchlichkeit wird durch verschiedene Längsschnittstudien zum so genannten Sommerloch verstärkt. Entwistle und Alexander (1992) beispielsweise haben in Baltimore Kinder von der Einschulung bis zur Highschool jeweils vor und nach der Sommerpause getestet. Dabei zeigte sich, dass ihre Leistungskurven während der Schulzeit parallel verliefen und erst in den langen Sommerferien auseinanderdrifteten. Eine von Baumert et al. (Limbird \& Stanat, 2006) durchgeführte Berliner-Studie belegt diesen Sommerloch-Effekt auch für die weit kürzeren deutschen Sommerferien: Kinder aus sozial benachteiligten Schichten und aus Zuwandererfamilien lernten in dieser Zeit weniger dazu als ihre Schulkameraden aus begüterteren Familien. Zwar scheinen solche Sommerlocheffekte Schereneffekte zu relativieren. Andererseits steht eine Vielzahl von Studien zur Verfügung, welche Matthäuseffekte im Sinne unterschiedlicher Lernzuwachse, insbesondere im sprachlichen und mathematischen Bereich, nachweisen. Diese Tatsache führt im nächsten Kapitel zu einer Diskussion von Matthäuseffekten in Förderprogrammen mit Kompensationscharakter, deren eigentliches Ziel somit darin besteht, solche Schereneffekte zu überwinden. 


\section{Matthäuseffekte in Förderprogrammen}

Im Allgemeinen basieren Förderprogramme auf der Annahme, dass Schereneffekte zwar eine Folge kognitiver Entwicklung und fehlenden Vorwissens sind, jedoch durch kompensatorische Einflüsse kontrolliert werden können. Allerdings erweist sich diese Annahme bei genauerem Hinsehen als problematisch. Aktuelle Förderprogramme haben häufig den überraschenden Effekt, dass sie Unterschiede zwischen leistungsstarken und leistungsschwachen Schülerinnen und Schülern nicht wie intendiert verkleinern, sondern eher vergrössern. Empirische Hinweise finden sich in vielen Studien, so etwa in den Untersuchungen von Deci und Papierno (2005) oder Bakermans-Kranenburg, van IJzendoorn und Bradley (2005), jedoch auch in deutschsprachigen Studien (Moser, Bayer \& Berweger, 2008; Stamm, 2006). Solche Matthäuseffekte lassen sich anhand von zwei alternativen Erklärungsmustern - einem leistungs- und einem zugangsbasierten - darstellen. Um leistungsbasierte Gewinne handelt es sich dann, wenn alle Schülerinnen und Schüler an einer Intervention partizipieren, aber die Leistungsstarken am deutlichsten davon profitieren. Nutzungsbasiert sind die Gewinne allerdings dann, wenn eine bestimmte Gruppe von einem Angebot aufgrund ihres besseren Zugangs, ihrer grösseren Motivation oder ihrer besseren sozialen oder ökonomischen Ressourcen im Vergleich zu einer benachteiligten Gruppe disproportional profitiert.

\section{Leistungsbasierte Matthäuseffekte}

Hinweise für leistungsbasierte Matthäuseffekte finden sich in vielen Bereichen. Besonders augenfällig sind sie in der Begabungsforschung, aber auch in der Forschung zur integrativen Förderung von fremdsprachigen benachteiligten Kindern. Als exemplarisches Beispiel dient ein Schweizer Sprachförderprojekt, das zwischen 2003 und 2005 in einer Gemeinde mit mehr als 48\% ausländischer Wohnbevölkerung aus über 70 Nationen durchgeführt wurde (Stamm, 2006). Im Kern war es zwar auf fremdsprachige, benachteiligte Kinder ausgerichtet, zielte jedoch explizit ebenso auf die Förderung einheimischer Kinder und auf Kinder ohne Sprachprobleme. Die kompensatorische Förderung umfasste Massnahmen wie Stützkurse (zwei Lektionen pro Woche), einen Deutsch-Intensivkurs (vier bis sechs Lektionen pro Woche) für neu eingewanderte Kinder sowie die intensive Individualisierung des sprachlichen Unterrichts für alle Kinder. Im Mittelpunkt der zwei Jahre dauernden wissenschaftlichen Begleitung mit zwei Messzeitpunkten in den Jahren 2004 und 2005 stand dabei die Frage, ob sich die Leistungen der anvisierten Kindergruppe verbesserten und ob dieses Modell somit eine Erfolg versprechende Antwort auf die Situation einer Schule mit einem hohen Anteil von Kindern mit Migrationshintergrund und mit einer grossen sprachlichen, sozialen und kulturellen Heterogenität sein kann.

In Abbildung 1 und 2 sind die Ergebnisse der insgesamt $n=315$ teilnehmenden Kinder dargestellt, von denen $\mathrm{n}=210$ fremdsprachige Kinder mit Migra- 
tionshintergrund und $\mathrm{n}=165$ einheimische Kinder waren. Dargestellt werden die Leistungen in den Fächern Deutsch und Mathematik. Sie wurden mit dem Testsystem Klassencockpit erhoben, einem vom Kanton St. Gallen zur Qualitätssicherung im Volksschulbereich entwickelten Instrumentarium. Die Leistungen der Schülerinnen und Schüler wurden im gängigen Notensystem erfasst und anschliessend in z-Werte transformiert. Der Migrationshintergrund wurde über die Lehrerangaben erhoben.

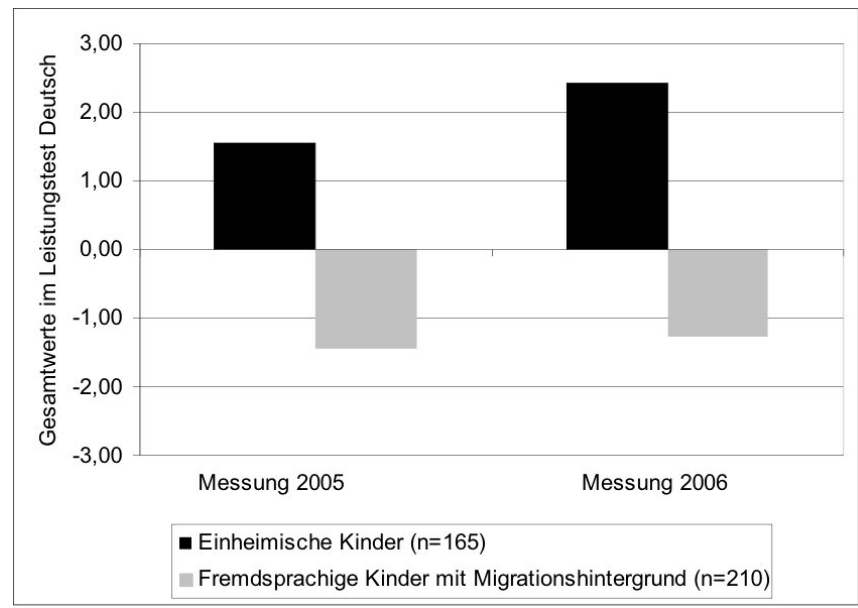

Abbildung 1: Gesamtwerte des Leistungstests Deutsch von fremdsprachigen Kindern mit Migrationshintergrund und einheimischen Kindern bei Beginn der Intervention und nach 12 Monaten (nach Stamm, 2006)

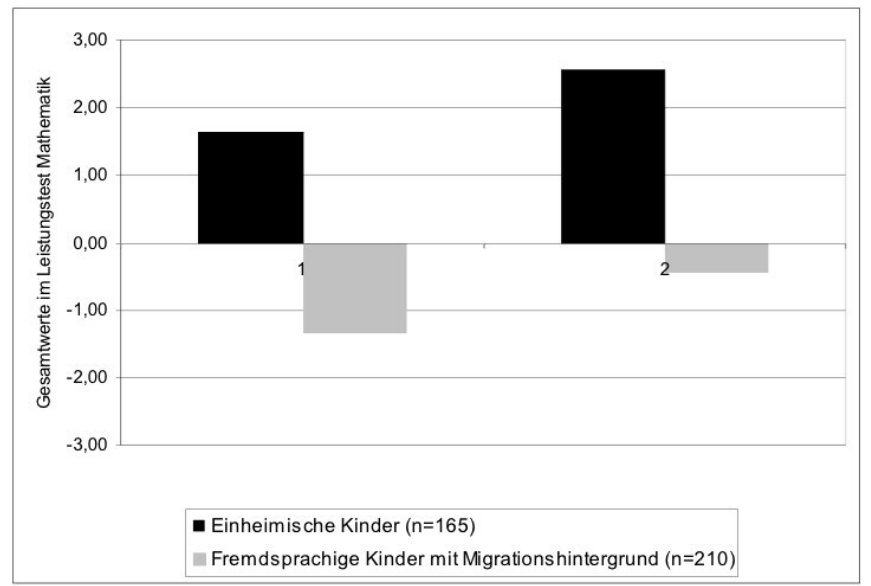

Abbildung 2: Gesamtwerte des Leistungstests Mathematik von fremdsprachigen Kindern mit Migrationshintergrund und einheimischen Kindern bei Beginn der Intervention und nach 12 Monaten (nach Stamm, 2006) 
Die Befunde in Abbildung 1 und 2 verdeutlichen, dass sich fremdsprachige Kinder mit Migrationshintergrund zu Beginn der Intervention in den Leistungstestergebnissen Deutsch signifikant zu Gunsten der bildungsnah aufwachsenden Kinder unterschieden $(t(315)=5.54, \mathrm{p}<.01)$. Etwas weniger deutlich, jedoch immer noch signifikant, fielen die Ergebnisse für den Leistungstest Mathematik aus $(t(314)=4.35, \mathrm{p}<.03)$. Besonders augenfällig ist, dass sich diese Unterschiede in beiden Kompetenzbereichen während der anderthalbjährigen Intervention vergrösserten (Deutsch: $t(302)=6.08, \quad \mathrm{p}<.01$ : Mathematik: $t(309)=5.14, \mathrm{p}<.03)$. Zwar zeigten die fremdsprachigen Kinder nach zwölf Monaten deutlich bessere Leistungen und entsprachen damit den intendierten Projektzielen, aber die Leistungen der privilegierten Kinder waren um ein Vielfaches besser, so dass sich die Unterschiede insgesamt weiter vergrösserten. Erstaunlicherweise galt dies sowohl für Deutsch als auch für Mathematik.

Einen ähnlichen Befund liefert die Evaluation des neuen Schweizer Schuleingangsmodells der Grund- und Basisstufe. Es vereint Kindergarten und erste Klasse ("Grundstufe») respektive Kindergarten sowie erste und zweite Klasse («Basisstufe»). Eines der Hauptziele dieses Fördermodells liegt darin, die ungleichen Startchancen privilegierter und benachteiligter Kinder durch die besondere Förderung Letzterer zu egalisieren. Die Evaluationsergebnisse von Moser et al. aus dem Jahr 2008 belegen anhand einer Stichprobe von $\mathrm{n}=934$ Kindern, dass sich die Unterschiede bisher nicht beseitigen liessen. Basis bildete das Testinstrumentarium wortgewandt und zablenstark (Moser \& Berweger, 2007), das sowohl sprachliche als auch mathematische Aufgaben enthält. Die Ergebnisse dieser Tests wurden in die standardisierte Normalverteilung transformiert und der Mittelwert zum ersten Testzeitpunkt auf 500 Punkte und die Standardabweichung auf 100 Punkte festgelegt. Der sozioökonomische Hintergrund wurde über einen Elternfragebogen erfasst. Ein hoher sozioökonomischer Status («SES hoch») umfasste Kinder mit privilegiertem Status, die Gruppe «SES mittel» solche mit eher privilegiertem oder eher benachteiligendem Status und die Gruppe mit tiefem sozioökonomischem Status («SES tief») Bislang liegen drei Messzeitpunkte vor $(2004,2006,2007)$. 


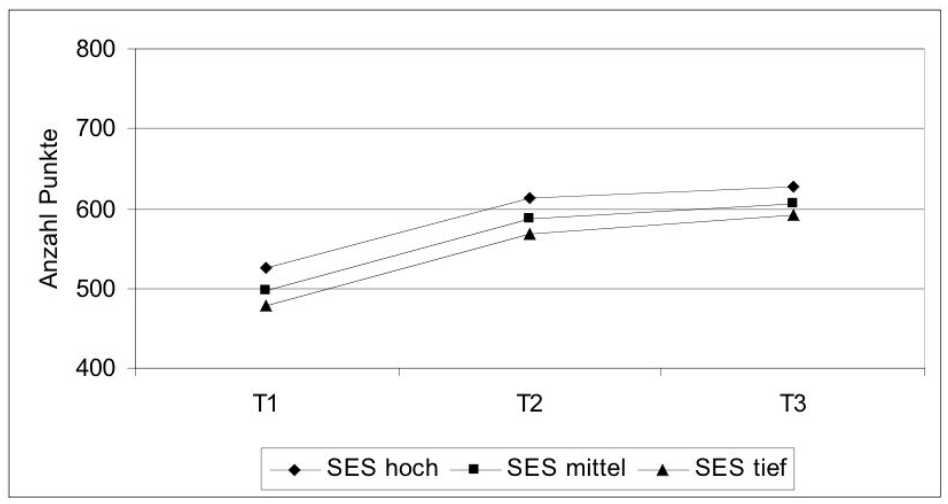

Abbildung 3: Lernfortschritte im Wortschatz nach sozialer Herkunft in der Grund-und Basisstufe in den ersten 36 Monaten (Moser et al., 2008, S. 52)

Abbildung 3 visualisiert die Leistungsfortschritte der Kinder, sortiert nach Wortschatz und sozialer Herkunft. Die Abbildung verdeutlicht, dass Kinder mit hohem SES nicht nur einen statistisch signifikant höheren Ausgangsmittelwert als Kinder mit tiefem SES hatten, sondern auch, dass die Vorteile der ersten Gruppe bis zum dritten Erhebungszeitpunkt stabil blieben und benachteiligte Kinder ihren Rückstand nach zwei Jahren (was ungefähr dem Schuleintritt entsprechen würde) nicht kompensieren konnten. Die Unterschiede zeigten sich am deutlichsten im in dieser Abbildung visualisierten Wortschatz, etwas weniger ausgeprägt bei der phonologischen Bewusstheit (als der Fähigkeit die Aufmerksamkeit auf die formalen Eigenschaften der gesprochenen Sprache zu lenken) sowie in Mathematik.

Aus diesen Befunden lässt sich schliessen, dass das neue Schuleingangsmodell eines seiner vielfach formulierten Ziele, Startchancengleichheit für alle Kinder zu schaffen, bisher nicht in erwünschtem Ausmass erreicht hat. Einer der Gründe dürfte darin liegen, dass Kinder mit tiefem SES zwar auch bedeutsame Fortschritte erzielten, Kinder mit hohem SES jedoch vom pädagogischen Modellkonzept möglicherweise aufgrund ihres Vorwissens, ihrer elaborierteren Lernstrategien und dem ausgeprägt bildungsorientierten Familienhintergrund etc. stärker profitierten. Damit verweisen diese Evaluationsbefunde nicht nur auf die enormen Schwierigkeiten, die Stabilität interindividueller Unterschiede möglichst früh aufzulösen, sondern ebenso auf die Problematik, solche Zielsetzungen überhaupt zu formulieren.

Fast identische Befunde liefert die deutsche Studie «BeLesen» zur integrativen Förderung von fremdsprachigen Kindern, in der Merkens et al. (2006) die geringe Beeinflussbarkeit der Lese- und Selbstkompetenzentwicklung von Migrantenkindern im Gegensatz zum deutlich grösseren Schulerfolg einheimischer Kinder nachweisen. Leistungsbasierte Effekte lassen sich jedoch auch in Förder- 
programmen für Hochbegabte finden. Aus der G8-Studie zur Begabtenförderung am Gymnasium von Heller (2002) wissen wir beispielsweise, dass überdurchschnittlich Begabte von individualisierenden und die Selbstverantwortung fördernden Unterrichtsmassnahmen weit stärker profitierten als durchschnittlich Begabte. Fast identische Befunde berichten Scruggs und Mastropierdi (1988) über ein Training zum Strategieerwerb, in welchem hochbegabte die nicht hochbegabten Jugendlichen - für die das Programm eigentlich gedacht war - im Hinblick auf den Wissenstransfer und die angewandten Strategiekonzepte deutlich übertrafen.

Allerdings liegen auch Befunde für positive Effektivitätsbilanzen vor. So belegen Paris und Jacobs (1984), dass nicht nur die leistungsstarken Schülerinnen und Schüler von einer Förderung profitieren müssen. Anhand der Ergebnisse zu ihrem Konzept der Förderung von Lesestrategien konnten sie nachweisen, dass leistungsschwache Kinder bei geeignetem metakognitivem Training genauso profitieren können. Ein weiteres Beispiel für solche Erfahrungen ist das Training zur Textverarbeitung von Hasselhorn und Körkel $(1984,1986)$ oder Jertz (2002).

Matthäuseffekte können sich auch zeigen, wenn Kinder unterschiedlichen Alters in identischen Angeboten gefördert werden. Aus Studien zum altersgemischten Lernen wissen wir, dass jahrgangsheterogener Unterricht mit dem Problem konfrontiert ist, dass die ältesten Schüler am meisten profitieren und die jüngeren überflügeln (Gutiérrez \& Slavin, 1992; Kucharz \& Wagener, 2007). Deshalb werden häufig auch leistungshomogene Gruppen gebildet. Gleiches zeigt sich in Interventionsstudien, wenn sie nach sozialem Hintergrund aufschlüsselt werden. Dass Kinder und Jugendliche aus bildungsnahen Familien disproportional von Interventionen profitieren, wenn sie generalisiert werden, zeigt sich in verschiedenen Studien zur Einschulungs- und Selektionspraxis (Avenarius et al., 2003; Gomolla \& Radtke, 2002) oder zu Trainingsprogrammen in der Berufsfindung (Imdorf, 2005).

Für den Bereich frühkindlicher Förderprogramme gibt es allerdings massiv gegenteilige Befunde. So weisen Studien von Magnuson, Ruhm und Waldfogel (2004), Spiess, Büchel und Wagner (2003), Sylva, Melhuish, Sammons, SirajBlatchford und Taggart (2008) oder Wolter und Coradi Vellacott (2003) nach, dass benachteiligt aufwachsende Kinder in qualitativ hochstehenden Programmen am deutlichsten profitieren, während die Gewinne für privilegiert aufwachsende Kinder eher bescheiden sind. Einer der Gründe dürfte darin liegen, dass eine hohe Angebotsqualität bei benachteiligten Kindern die möglicherweise negativen Auswirkungen familiärer Einflussmerkmale kompensieren kann, währenddem die Qualität des häuslichen Umfelds privilegiert aufwachsender Kinder entwicklungsförderlicher ist und das Vorschulangebot deshalb kein Kompensationsgut darstellt. 


\section{Zugangsbasierte Matthäuseffekte}

In den letzten Jahren sind viele Angebote aufgebaut worden, die als niederschwellig organisierte Interventionen Nutzungshindernisse abbauen sollen. Dazu gehören beispielsweise Elternbildungsprogramme (Bakermans-Kranenburg et al., 2005; Deutscher Familienverband, 2009; Kast, 2006), Nachhilfeprogramme (Dohmen, Erbes, Fuchs \& Günzel, 2008), Sommerakademien (Cooper, Charlton, Valentine \& Muhlenbruck, 2000) oder auch Lehrevaluationen (Rindermann, 2001). Solche Programme wären im Kern zwar meist auf die Adressatengruppen ausgerichtet, für die sie entwickelt worden sind (Eltern aus bescheidenen sozialen Verhältnissen oder anderer kultureller Angehörigkeit, leistungsschwache Schülerinnen und Schüler, Dozierende mit Lehrproblemen), doch werden sie aus politischen oder strategischen Gründen meist für alle Interessierten zugänglich gemacht. Gemäss Spoth, Redmond, Hockaday \& Shin (1996) dürfte in diesem freien Zugang einer der Gründe liegen, weshalb solche Angebote mit Matthäuseffekten verknüpft sein können. In der Evaluation eines Drogenprogramms, das an weiterführenden Schulen durchgeführt worden war, konnten sie nachweisen, dass besser situierte Familien von solchen Programmen stärker profitierten als benachteiligte Familien. Einer der Hauptgründe lag darin, dass diese benachteiligten Familien Bedenken hatten, ihre Anonymität würde gegenüber den anderen Familien nicht genug gewahrt. Ähnliche Befunde stammen aus der Fernsehserie Sesamstrasse. Sie ist in den letzten dreissig Jahren nicht nur zum Herzstück des öffentlichen Fernsehens geworden, sondern auch zu einer führenden Einflussgrösse in der (amerikanischen) Diskussion der medialen Wirkung auf junge Kinder. Ursprünglich war Sesamstrasse in den USA als Mittel zur Unterstützung von benachteiligen Vorschulkindern und ihren Familien konzipiert und auch evaluiert worden. Hatte man zunächst noch angenommen, dass häufiger Sesam-Konsum die kognitive kindliche Entwicklung fördere, so weisen neuere Analysen jedoch einhellig nach, dass es vor allem die Unterschiede in den Fernsehgewohnheiten zwischen privilegierten und eher benachteiligten Kindern sind, welche die intellektuellen Schereneffekte zwischen den beiden Gruppen zu Gunsten der privilegiert aufwachsenden Kinder vergrössern (Anderson, Huston, Schmitt, Linebarger \& Wright, 2001; Fisch, 2005).

Dass jedoch auch der im vorangehenden Kapitel dargestellte Befund, wonach benachteiligt aufwachsende Kinder in qualitativ hochstehenden Programmen am deutlichsten profitieren, nicht per se belegt eine Metaanalyse von Bakermans-Kranenburg et al. (2005). Tabelle 1 präsentiert die Hauptergebnisse. Ausgehend von der Frage, ob präventive frühkindliche Interventionsprogramme effektiv sind, untersuchten die Autoren 48 Aufsätze mit 56 Interventionseffekten bei N=7'350 Familien. In allen diesen Studien wurde die Effektivität auf der Basis der HOME Inventory Scale gemessen. Dabei handelt es sich um eines der am verbreitetsten Instrumentarien für die Messung der Qualität kindlicher Lernumgebungen, welches qualitative und quantitative Aspekte der Stimulation und der Unterstützung des Kindes durch seine Familie im häuslichen Umfeld misst. 36 
zufallsausgewählte Studien wurden in den USA durchgeführt, vier in anderen Ländern (Australien und Kanada).

Tabelle 1: Ergebnisse der Metaanalyse der Moderatoren in den HOMEGesamtwerten (Bakermans-Kranenburg et al., 2005, S. 12)

\begin{tabular}{|c|c|c|c|c|c|c|c|}
\hline & 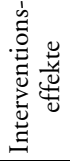 & $\mathrm{n}$ & d & $95 \% \mathrm{CI}$ & $\begin{array}{c}\mathrm{Q} \\
\text { Homoge- } \\
\text { neity }\end{array}$ & $\begin{array}{c}\mathrm{Q} \\
\text { Comparison }\end{array}$ & $\begin{array}{c}\mathrm{p} \\
\text { Comparison }\end{array}$ \\
\hline Total & 56 & 7350 & $0.20^{* *}$ & $(0.12 \sim 0.27)$ & $152.8^{* * *}$ & 23.0 & $<.001$ \\
\hline $\begin{array}{l}\text { Mothers } \\
\text { ethnicity }\end{array}$ & & & & & & 1.6 & .20 \\
\hline white & 17 & 1644 & $0.19^{*}$ & $(0.02-0.36)$ & $41.3^{* * *}$ & & \\
\hline non-white & 23 & 5002 & $0.10^{*}$ & $(0.02 \sim 0.19)$ & $38.3^{*}$ & & \\
\hline Country & 40 & & & & & 21.0 & $<.001$ \\
\hline U.S. & 36 & 6350 & $0.10^{*}$ & $(0.03 \sim 0.16)$ & $52.0^{*}$ & & \\
\hline Non-U.S & 4 & 296 & $0.52^{*}$ & & $8.3^{*}$ & & \\
\hline SES & & & & & & 3.7 & .05 \\
\hline Low & 36 & 5705 & $0.12^{*}$ & $(0.03 \sim 0.20)$ & $74.4^{* *}$ & & \\
\hline Middle & 4 & 941 & $0.25^{* * *}$ & $(0.10 \sim 0.40)$ & 3.1 & & \\
\hline Adolescent & & & & & & 17.4 & $<.001$ \\
\hline$>70 \%$ & 11 & 1269 & 0.11 & $(-0.00 \sim 0.23)$ & & & 0.11 \\
\hline $30 \%-70 \%$ & 10 & 1926 & -0.02 & $(-0.11 \sim 0.07)$ & 6.4 & & \\
\hline$<30 \%$ & 19 & 3451 & $0.24^{* * *}$ & $(0.10 \sim 0.37)$ & $48.7^{* * *}$ & & \\
\hline
\end{tabular}

${ }^{*} \mathrm{p}<.05 ;{ }^{* *} \mathrm{p}<.01 ; \mathrm{p}<.001$

Während die in diesen Ländern durchgeführten Studien substantielle Effektgrössen von $\mathrm{d}=0.52$ zeigten, betrugen sie in den USA-Studien lediglich 0.10 . Der Kontrast war signifikant $(\mathrm{Q}=21.0, \mathrm{p}<.001)$. In 17 Studien bestanden die Stichproben vorwiegend aus weissen Probanden. In diesen Studien war die kombinierte Effektgrösse $(\mathrm{d}=0.19)$ vergleichbar mit der kombinierten Effektgrösse für das Subsample der nicht weissen Teilnehmenden ( $d=0.10 ; \mathrm{Q}=1.6, \mathrm{p}=.20)$. Stichproben mit tiefem SES schienen weniger von den Interventionen zu profitieren als Mittelschichtstichproben $(\mathrm{d}=0.25 ; \mathrm{Q}=3.7, \mathrm{p}=.05)$. Gleiches zeigte sich in Studien mit adoleszenten Müttern. Sie alle wiesen tiefere Effektgrössen auf ( $>70 \%$ adolescent: $\mathrm{d}=0.11 ; 30-70 \%$ adolescent: $\mathrm{d}=-0.02$ ) als Studien mit weissen oder ohne respektive wenigen adoleszenten Müttern $(\mathrm{d}=0.24 ; \mathrm{Q}=17.4, \mathrm{p}$ $<.001)$. Damit deuten diese Befunde darauf hin, dass Mütter aus benachteiligten Sozialschichten solchen Angeboten gegenüber deutlich zurückhaltender waren als Mütter aus privilegierteren Milieus. Weisse, über dreissigjährige Mittelschicht-Mütter profitierten am meisten vom Interventionsangebot. Diese Befunde unterstützen somit den Matthäuseffekt: Gut situierte Mütter nutzen früh- 
kindliche Interventionsangebote stärker und profitieren auch ausgeprägter von ihnen als diejenigen, welche am meisten benachteiligt sind. Damit tragen solche Angebote möglicherweise ungewollt zur Verstärkung bestehender Disparitäten bei.

Augenfällige Beispiele für zugangsbasierte Matthäuseffekte finden sich auch in Begabtenförderprogrammen. Sowohl mit Fokus auf den deutschsprachigen (Imhasly, 2004; Stamm, 2001) als auch auf den anglo-amerikanischen Raum (Benbow \& Stanley, 1996; Donovan \& Cross, 2002) zeigt sich durchgehend das gleiche Bild: Förderprogramme werden zwar explizit allen Schülerinnen und Schülern mit überdurchschnittlichen Begabungen angeboten. Trotzdem sind leistungsstarke und im Verhalten angepasste Kinder aus der Mittel- und Oberschicht deutlich übervertreten, solche aus bildungsfernen Milieus jedoch massiv unterrepräsentiert. Der Hauptgrund dürfte einerseits darin liegen, dass privilegierte Familien viel aktiver sind, wenn es darum geht, die kognitiven Fähigkeiten ihres Kindes abzuklären und Lehrkräfte dazu zu drängen, es in einem Förderprogramm zu platzieren. Andererseits tendieren bildungsferne Familien dazu, das Potenzial ihres Kindes zu unterschätzen und es nicht entsprechend zu wertschätzen. Ähnliches gilt für Lehrkräfte, wenn sie einen hohen Bildungsgrad sowohl als Indikator für die bessere genetische Ausstattung als auch für die Erklärung, weshalb Schülerinnen und Schüler aus benachteiligten Milieus in Begabtenförderprogrammen quasi inexistent seien. Insgesamt hat die Übervertretung privilegiert aufwachsender Kinder in Förderprogrammen zu kritischen Kommentaren geführt, wonach Begabtenförderung ihr Ziel verfehlt habe, weil sie die bestehenden sozialen Ungleichheiten verstärke und die soziale Vererbungspraxis zementiere (Giesinger, 2008; Stamm, 2009).

Somit verweist auch der diesem Kapitel zu Grunde gelegte spezifische Blick auf leistungs- und zugangsbasierte Matthäuseffekte in Förderprogrammen zwar auf einige Widersprüchlichkeiten, legitimiert in der Tendenz jedoch die Annahme, dass bestehende Disparitäten durch spezifische Fördermassnahmen selten ausgeglichen werden können, sondern eher verstärkt werden. Wenn somit leistungsstarke und/oder privilegierte Schülerinnen und Schüler besonders profitieren und ihre initialen Vorteile durch Fördermassnahmen multiplizieren können, dann stellt sich die Frage, wie mit Matthäuseffekten grundsätzlich umgegangen werden soll, wenn kompensatorische Massnahmen nicht die intendierten Effekte erzielen. Diese Frage wird im nächsten Kapitel diskutiert.

\section{Perspektiven des Diskurses von Matthäuseffekten}

Zunächst lässt sich unter Rekurrenz auf die soziale Ungleichheitsforschung und die Risikoforschung (vgl. Alheit, 1993; Blossfeld \& Shavit, 1993; Ditton, 2004; Hradil, 2001) die These formulieren, dass solche Angebote ausschliesslich schwächeren oder benachteiligten Gruppen angeboten werden sollten, damit die 
Effektivitätsbilanzen verbessert werden könnten. Diese These lässt sich aus unterschiedlichen Perspektiven diskutieren.

Auch aus politisch-ökonomischer Perspektive lassen sich Argumente formulieren, die eine Generalisierung unterstützen. Gemäss Heckman, Stixrud und Urzua (2006) ist die Position eines Landes im Zuge der Globalisierung und des internationalen Wettbewerbs verstärkt von seinen Top-Wissenschaftlern, Politikern, Schriftstellern, Ingenieuren etc. abhängig. Diese wiederum rekrutieren sich bevorzugt aus dem Spitzenperzentil der Schülerpopulation. Deshalb steigert jede Intervention, welche die Leistungen der besten Schülerinnen und Schüler positiv beeinflusst, auch die internationale Wettbewerbsfähigkeit eines Landes. Gerade im Zuge der PISA-Studie konnte jedoch nachgewiesen werden, dass trotz erheblichem Mittelaufwand die Leistungsspitze in allen untersuchten Domänen relativ klein ist (Zimmer, Brunner, Lüdtke, Prenzel \& Baumert, 2007). So betrug der Schüleranteil in der höchsten Mathematik-Kompetenzstufe in Ländern des Mittelfeldes wie Deutschland oder Österreich je 4.0\% und in der Schweiz 6.0\%, währenddem er in Ländern der Spitzengruppe deutlich höher war und in Südkorea bei 8.1\% oder in den Niederlanden bei 7.3\% lag (Deutsches PISA-Konsortium, 2002). Auffallend ist dabei, dass in diesen Ländern auch die Anteile der Risikogruppen kleiner sind (Finnland 6.0\%, Südkorea 9.0\%, Niederlande 1.0\%). Es werden aber auch Argumente ins Feld geführt, dass eine Verringerung der Leistungsunterschiede ebenso wichtig sei für die zukünftige Wettbewerbsfähigkeit eines Landes. Dabei steht meist die ca. 15\% bis 20\% umfassende Risikogruppe (Stanat \& Schneider, 2002) im Zentrum, die grosse mediale Beachtung gefunden hat und auch in der Wissenschaft ausführlich diskutiert worden ist (Becker \& Lauterbach, 2004). Bildungsökonomische Analysen wie die von Carniero und Heckman (2002) gehen davon aus, dass mittels einer bereits im Vorschulalter einsetzenden Förderung und einer nachfolgend auf ein Minimalniveau angehobenen Leistungsbilanz der Risikogruppen zukünftige Sozialausgaben verringert und ein günstigeres Kosten-Nutzen-Verhältnis generiert werden könnte. Darauf verweisen auch zahlreiche Längsschnittstudien zur frühkindlichen Förderung. Ist die Betreuungsqualität und das Ausbildungsniveau des Personals hoch, dann sind damit langfristige, positive Kompensationseffekte auf den späteren Schulerfolg, die Bildungslaufbahn und auch auf die soziale und gesundheitliche Entwicklung verbunden, die mit einer Reduktion von Sozialleistungen, Kriminalität, Klassenwiederholungen, Schulverweigerung und Schulabbruch einhergehen (Barnett, 2008).

Im Ergebnis sind somit alle diese Perspektiven mit dem Paradigma der Chancengerechtigkeit kompatibel. Wenn die Gesellschaft die Optimierung der Leistungen aller Kinder, der leistungsschwachen und der leistungsstarken, als Ziel erachtet und Förderangebote allen zugänglich gemacht werden sollen, dann bereiten disproportionale Leistungsgewinne der Befähigteren und Privilegierteren keine besonderen Probleme. Werden Fördermassnahmen jedoch mit Zielen verbunden, die ungleichen Startchancen privilegierter und benachteiligter Kinder 
durch die spezifische Kompetenzförderung letzterer zu egalisieren, dann sind fokussiertere Anstrengungen nötig.

\section{Pädagogische Konsequenzen}

Die in diesem Aufsatz empirisch belegte Tatsache, dass viele Förderprojekte den überraschenden Effekt haben, bestehende Unterschiede zwischen leistungsschwachen und leistungsstarken Aufwachsenden dann im Sinne von Matthäuseffekten zu verstärken, wenn sie allen Kindern und Jugendlichen angeboten werden, ist von der Wissenschaft zwar verschiedentlich untersucht und auch von der und Bildungspolitik beachtet worden, doch haben sich die Diskussionen vor allem auf system- oder lernpsychologisch bedingte Erklärungsmuster konzentriert (Heller, 2004). Wie mit diesem problematischen Phänomen umgegangen - d.h. ob und wie es am besten reduziert werden sollte oder könnte - ist bislang jedoch selten reflektiert worden. Abschliessend wird deshalb versucht, einige pädagogische Konsequenzen zu formulieren, die sich am Taxonomiemodell und seinen leistungs- und zugangsorientierten Gewinnen orientieren.

Im Hinblick auf die Reduktion leistungsbasierter Gewinne können Optimierungsmöglichkeiten vor allem in der didaktischen Standardantwort auf steigende Heterogenität von Hentigs «Die Menschen stärken, die Sachen klären» (1985) gesucht werden. Optimierungsmöglichkeiten legitimieren sich vor dem Hintergrund zahlreicher Analysen zu den unterschiedlichen Bilanzen der an der PISA-Studie beteiligten Staaten. So zeigt Willms (2006) auf, dass die erfolgreichsten Länder diejenigen mit den kleinsten Raten leistungsschwacher Schülerinnen und Schüler sind und die grösste Varianz innerhalb der Schulen und nicht zwischen ihnen liegen. Aus der Studie COACTIV der Forschergruppe um Baumert et al. (Brunner et al., 2006) zur Bedeutung der Fachdidaktik wissen wir, dass Mathematiklehrkräfte, die über ein breites fachliches und fachdidaktisches Repertoire verfügen, besser helfen können und ihrer Schülerinnen und Schüler zu deutlich besseren Lernfortschritten führen. Gut eingesetztes fachdidaktisches Wissen führt somit zu grösseren Lernerfolgen als Vorwissen allein. Um «die Sachen [zu] klären», müssen didaktische und fachdidaktische Bemühungen auf die Hebung des Leistungsniveaus fokussieren und Lerntempo sowie Anspruchsniveau dem kognitiven Aktivierungspotenzial angepasst werden. Dazu ist jedoch diagnostische Kompetenz sowie pädagogisch-psychologisches Wissen über die Gestaltung und Optimierung von Lernsituationen nötig: Dazu gehören Wissen über Lern- und Leseschwächen, über Hochbegabung, Aufmerksamkeitsprobleme und Klassenzimmermanagement. «Die Menschen stärken» basiert auf der Erkenntnis, dass Lernen ein hochgradig einzigartiger Prozess ist, für den kollektive Lernphasen über weite Strecken ungeeignet sind. Notwendig sind deshalb hohe Lehrererwartungen und Individualisierungsbemühungen, das sich an unterschiedlichen Adressatengruppen flexibel orientieren und die Stärkung des 
einzelnen Schülers zum Ziel haben.

Zugangsbasierte Gewinne können durch eine frühe und systematische Förderung egalisiert werden. Diese Art von Förderung legitimiert sich aus der Tatsache, dass es nicht einzelne Indikatoren sind, sondern komplexe Wechselwirkungen, welche zu Schereneffekten führen. Insofern würde auch eine ausschliesslich auf frühe Sprachförderung fokussierte Förderung zu kurz greifen. Eine solche Frühförderung müsste zudem ein komplexes und variantenreiches Angebot beinhalten, das die Eltern so einbezieht, damit ihre Erziehungskompetenz gestärkt wird und dass sie niederschwellige Zugangsmöglichkeiten zu Förderprogrammen, zu ausserschulischer Unterstützung und Sommerlernprogrammen haben.

Letztlich liefern die PISA-Studien neue Erkenntnisse, welche die Diskussion des Umgangs mit Matthäuseffekten bereichern. Weil es erfolgreiche Länder gibt, welche nicht nur die Leistungen ihrer Schülerinnen und Schüler aus weniger bevorzugten Familien stützen, sondern auch in der Egalisierung der Leistungsunterschiede Spitzenplätze belegen, dann liefern sie deutliche Hinweise dafür, dass gute Schulleistungen und Chancengerechtigkeit Hand in Hand gehen können. Die Frage, die Gardner (1961) vor einem halben Jahrhundert gestellt hat, lässt sich auf dieser Folie positiv beantworten: «Excellence: Can we be equal and excellent too?»

\section{Literaturverzeichnis}

Aarnoutse, C. A. J. \& van Leeuwe, J. F. J. (2000). Development of poor and better readers during the primary school. Educational Research and Evaluation, 6, 251-278.

Alheit, P. (1993). Ambivalenz von Bildung in modernen Gesellschaften: Strukturprinzip kumulativer Ungleichheit oder Potential biographischer Handlungsautonomie? In A. Meier \& U. Rabe-Kleberg (Hrsg.), Weiterbildung, Lebenslauf, sozialer Wandel. (S. 87-103). Berlin: Luchterhand.

Anderson, D. R., Huston, A. C., Schmitt, K. L., Linebarger, D. L. \& Wright, J. C. (2001). Early childhood television viewing and adolescent behavior. Monographs of the Society for Research in Child Development, 66 (1), 1-180.

Avenarius, H., Ditton, H., Döbert, H., Klemm, K., Klieme, E., Rürup, M., Tenorth, H.-E., Weishaupt, H. \& Weiss, M. (2003). Bildungsbericht Deutschland: Erste Befunde. Opladen: Leske und Budrich.

Baird, L. L. (1985). Do grades and tests predict adult accomplishment? Research in Higher Education, 23, 3-85.

Bakermans-Kranenburg, M. J., van IJzendoorn, M. H. \& Bradley, R. H. (2005). Those who have, receive: The Matthew effect in early childhood intervention in the home environment. Review of Educational Research, 75 (1), 1-26.

Barnett, S. W. (2008). Preschool education and its lasting effects: Research and policy implications. Retrieved Oktober 5, 2010, from http://epicpolicy.org/publication/preschool-education

Barz,H. \& Tippelt, R. (Hrsg.) (2004). Weiterbildung und soziale Milieus in Deutschland. Band 2: Adressaten- und Milieufoschung zu Weiterbildungsverhalten und -interessen. Bielefeld: Bertelsmann.

Bast, J. \& Reitsma, P. (1998). Analyzing the development of individual differences in terms of Matthew effects: Results from a Dutch longitudinal study. Developmental Psychology, 34, 1373-1399. 
Baumert, J., Stanat, P. \& Watermann, R. (2006). Schulstruktur und die Entstehung differenzieller Lern- und Entwicklungsmilieus. In J. Baumert et al. (Hrsg.), Herkunftsbedingte Disparitäten im Bildungswesen: Differenzielle Bildungsprozesse und Probleme der Verteilungsgerechtigkeit. Vertiefende Analysen im Rahmen von PISA 2000 (S. 95-188). Wiesbaden: VS Verlag für Sozialwissenschaften.

Becker, M., Lüdtke, O., Trautwein, U. \& Baumert, J. (2006). Leistungszuwachs in Mathematik : Evidenz für einen Schereneffekt im mehrgliedrigen Schulsystem? Zeitschrift für Pädagogische Psychologie, 20 (4), 233-242.

Becker, W. \& Lauterbach, R. (2004). Dauerhafte Bildungsungleichheiten - Ursachen, Massnahmen, Prozesse und Wirkungen. In W. Becker \& H. Lauterbach (Hrsg.), Bildung als Privileg? Erklärungen und Befunde zu den Ursachen der Bildungsungleichheit (S. 9-40). Wiesbaden: VS Verlag für Sozialwissenschaften.

Benbow, C. P. \& Stanley, J. C. (1996). Inequity in equity: How current educational equity policies place able students at risk. Psychology, Public Policy, and Law, 2, 249-293.

Bessoth, R. (1994). Lehrerberatung - Lehrerbeurteilung. Berlin: Luchterhand.

Blossfeld, H.-P. \& Shavit, Y. (1993). Dauerhafte Ungleichheiten. Zur Veränderung des Einflusses der sozialen Herkunft auf die Bildungschancen in dreizehn industrialisierten Ländern. Zeitschrift für Pädagogik, 39 (1), 25-52.

Brunner, M., Kunter, M., Krauss, S., Klusmann, U., Baumert, J., Blum, W., Neubrand, M., Dubberke, T., Jordan, A., Löwen, K. \& Tsai. Y.-M. (2006). Die professionelle Kompetenz von Mathematiklehrkräften: Konzeptualisierung, Erfassung und Bedeutung für den Unterricht. Eine Zwischenbilanz des COACTIV-Projekts. In M. Prenzel \& L. Allolio-Näcke (Hrsg.), Untersuchungen zur Bildungsqualität von Schule. Abschlussbericht des DFG Schwerpunktprogramms (S. 54-83). Münster: Waxmann.

Carniero, P. \& Heckman, J. J. (2002). Human capital policy. Paper presented at the Alvin Hausen Seminar. Cambridge, MA: Harvard University.

Chi, M. T. H., Glaser, R. \& Rees, E. (1982). Expertise in problem solving. In R. Sternberg (Ed.), Advances in the psychology of human intelligence 1, (pp. 7-76). Hillsdale, NJ: Erlbaum.

Cooper, H., Charlton, K., Valentine, J. C. \& Muhlenbruck, L. (2000). Making the most of summer school: A meta-analytic and narrative review. Monographs of the Society for Research in Child Development, 65 (1, Serial No. 260), 1-180.

Deci, S. J. \& Papierno, P. B. (2005). The rhetoric and reality of gap closing. When the «HaveNots» gain but the "Haves» gain even more. American Psychologist, 60 (2), 149-160.

Deutscher Familienverband. (Hrsg.). (2009). Handbuch Elternbildung. Opladen: Leske und Budrich.

Deutsches PISA-Konsortium. (2002). PISA 2000 - Die Länder der Bundesrepublik Deutschland im Vergleich. Opladen: Leske und Budrich.

Ditton, H. (2004). Schule und sozial-regionale Ungleichheit. In W. Helsper \& J. Böhme (Hrsg.), Handbuch der Schulforschung (S. 605-624). Opladen: Leske und Budrich.

Dohmen, D., Erbes, A., Fuchs, K. \& Günzel, A. (2008). Was wissen wir über Nachhilfe? Sachstand und Auswertung der Forschungsliteratur zu Angebot, Nachfrage und Wirkungen. Berlin: Bundesministeriums für Bildung und Forschung.

Donovan, M. S. \& Cross, C. T. (Ed.). (2002). Minority students in special and gifted education. Washington: National Research Council.

Entwistle, D. \& Alexander, K. (1992). Summer setback: Race, poverty, school composition and educational stratification in the United States. American Sociological Review, 57, 72 84.

Fisch, S. (2005). Children's learning from television. It is not just violence. Televizion, 18, 1014.

Gardner, J. W. (1961). Excellence: Can we be equal and excellent too? New York: Harper and Row. 
Giesinger, J. (2008). Begabtenförderung und Bildungsgerechtigkeit. In H. Ullrich \& S. Strunk (Hrsg.), Begabtenförderung an Gymnasien. Entwicklungen, Befunde, Perspektiven (S. 271-291). Wiesbaden: VS Verlag für Sozialwissenschaften.

Gomolla, M. \& Radtke, F.-O. (2002). Institutionelle Diskriminierung. Die Herstellung ethnischer Differenz in der Schule. Opladen: Leske und Budrich.

Gruber, E. (2001). Beruf und Bildung - (k)ein Widerspruch? Bildung und Weiterbildung in Modernisierungsprozessen. Innsbruck: Studienverlag.

Gutiérrez, R. \& Slavin, R. E. (1992). Achievement effects of non-graded elementary schools: A best evidence synthesis. Review of Educational Research, 62 (4), 333-334.

Hasselhorn, M. \& Körkel, J. (1984). Zur differentiellen Leistung metakognitiver Komponenten für das Verstehen und Behalten von Texten. Zeitschrift für Entwicklungspsychologie und Pädagogische Psychologie, 16, 283-296.

Hasselhorn, M. \& Körkel, J. (1986). Metacognitive versus traditional reading instructions: The mediating role of domain-specific knowledge on children's text processing. Human Learning, 5, 75-90.

Heckman, J. J., Stixrud, J. \& Urzua, S. (2006). The effects of cognitive and noncognitive abilities on labor market outcomes and social behavior. NBER Working Papers 12006, Cambridge, MA: National Bureau of Economic Research, Inc.

Heller, K.A. (2002). Begabtenförderung am Gymnasium. Ergebnisse einer zehnjährigen Längsschnittstudie. Opladen: Leske und Budrich.

Heller, K. A. (2004). Schullaufbahnentscheidung und Bildungserfolg. Mythen und Fakten. Profil, 12, 16-22.

Helmke, A. \& Weinert, F. E. (1987). Unterrichtsqualität und Leistungszuwachs. In MaxPlanck-Gesellschaft (Hrsg.), Jahrbuch 1986 (S. 821-823). München: Max-Planck-Gesellschaft.

Hradil, S. (2001). Soziale Ungleichheit in Deutschland. Opladen: Leske und Budrich.

Hulin, C. L., Henry, R. A. \& Noon, S. L. (1992). Adding a dimension: Time as a factor in the generalizability of predictive relationship. Psychological Bulletin, 107, 328-340.

Imdorf, Ch. (2005). Schulqualifikation und Berufsfindung. Wie Geschlecht und nationale Herkunft den Übergang in die Berufsbildung strukturieren. Wiesbaden: VS Verlag für Sozialwissenschaften.

Imhasly, M. (2004). Metaevaluation Begabungsförderung. Forschungsbereich Schulqualität und Schulentwicklung der Universität Zürich.

Jertz, J. (2002). Vermittlung allgemeiner, fachübergreifender Lernkompetenzen bei Regelschülern im Gymnasium - empirische Gruppenuntersuchung zur differentiellen Indikation zweier Förderprogramme. Dissertation, Universität Köln.

Kast, F. (2006). «Denn wer hat, dem wird (dazu) gegeben, und er wird im Überfluss haben ...» Bildungsschicht- und regionsspezifische Besuchsquoten des Gymnasiums (Sekundäranalyse der Volkszählungsdaten). Erziehung und Unterricht, 3-4, 236-263.

Köller, O. \& Baumert, J. (2002). Entwicklung schulischer Leistungen. In R. Oerter \& L. Montada (Hrsg.), Entwicklungspsychologie (S. 756-786). Weinheim: Beltz.

Kucharz, D. \& Wagener, M. (2007). Jahrgangsübergreifendes Lernen. Eine empirische Studie zu Lernen, Leistung und Interaktion von Kindern in der Schuleingangsphase. Hohengehren: Schneider.

Limbird, C. \& Stanat, P. (2006). Sprachförderung bei Schülerinnen und Schülern mit Migrationshintergrund: Ansätze und ihre Wirksamkeit. In J. Baumert et al. (Hrsg.), Herkunftsbedingte Disparitäten im Bildungswesen. Differenzielle Bildungsprozesse und Probleme der Verteilungsgerechtigkeit. Vertiefende Analyse im Rahmen von PISA 2000 (S. 257-307). Wiesbaden: VS Verlag für Sozialwissenschaften.

Luyten, H., Cremers-van Wees, L. M. \& Bosker, R. J. (2003). The Matthew effect in Dutch primary education, differences between school, cohorts and pupils. Research Papers in Edu- 
cation, 18 (2) 167-195.

Magnuson, K., Ruhm, C. J. \& Waldfogel, J. (2004). Does prekindergarten improve school preparation and performance? NBER Working Paper 10452. Cambridge, MA: National Bureau of Economic Research, Inc.

Merkens, H., Schründer-Lenzen, A., Bellin, N., Francke, J., Gelfort, K., Heintze, A. \& Mücke, S. (2006). Schriftspracherwerb bei Kindern mit Migrationshintergrund. Endbericht des Projekts BeLesen: Berliner Längsschnittstudie zur Lesekompetenzentwicklung von Grundschulkindern. Freie Universität Berlin.

Merton, R. K. (1968). The Matthew effect in science. Science, 159, 56-63.

Moser, U., Bayer, N. \& Berweger, S. (2008). Summative Evaluation Grundstufe und Basisstufe. Zwischenbericht zuhanden der EDK-Ost. Universität Zürich.

Moser, U. \& Berweger, S. (2007). wortgewandt \& zahlenstark. Zürich: Lehrmittelverlag.

Paris, S. G. \& Jacobs, J. E. (1984). The benefits of informed instruction for children's reading awareness and comprehension skills. Child Development, 55, 2083-2093.

Plomin, R., DeFries, J. C. \& Loehlin, J. C. (1977). Genotype-environment interaction and correlation in the analysis of human behavior. Psychological Bulletin, 84, 309-322.

Rindermann, R. (2001). Lehrevaluation. Einführung und Überblick zu Forschung und Praxis der Lehrveranstaltungsevaluation an Hochschulen mit einem Beitrag zur Evaluation computerbasierten Unterrichts. Landau: Verlag Empirische Pädagogik.

Schmidt, F. L., Hunter, J. E. \& Outerbridge, A. N. (1986). The joint relation of experience and ability with job performance: A test of three hypothesis. Journal of Applied Psychology, 62, 529-540.

Schneider, W. \& Stefanek, J. (2004). Entwicklungsveränderungen allgemeiner kognitiver Fähigkeiten und schulbezogener Fertigkeiten: Evidenz für einen Schereneffekt? Zeitschrift für Entwicklungspsychologie und Pädagogische Psychologie, 36, 147-159.

Scruggs, T. E. \& Mastropierdi, M. A. (1988). Acquistion and transfer of learning strageies of gifted and non gifted students. Journal of Special Education, 22, 153-166.

Shaywitz, B. A., Holford, T. R., Holahan, J. M., Fletcher, J. M., Stuebing, K. K. \& Francis, D. J. (1995). A Matthew effect for IQ but not for reading. Reading Research Quarterly, 30, 894-906.

Spiess, C. K., Büchel, F. \& Wagner, G. G. (2003). Children's school placement in Germany: Does Kindergarten attendance matter? Early Childhood Research Quarterly, 18 (2), 255270.

Spoth, R., Redmond, C., Hockaday, C. \& Shin, C. H. (1996). Barriers to participation in family skills preventive interventions and their evaluations: A replication and extension. $\mathrm{Fa}$ mily Relations, 45, 247-254.

Stamm, M. (2001). Begabungsförderung an den Zürcher Volksschulen. Eine Überprüfung der Pilotphase. Zürich: Schul- und Sportdepartement.

Stamm, M. (2005). Zwischen Exzellenz und Versagen. Schullaufbahnen von Früblesern und Frührechnerinnen. Chur: Rüegger.

Stamm, M. (2006). Evaluation des Sprachförderprojekts Spreitenbach. Detailanalysen. Universität Fribourg, Departement Erziehungswissenschaften.

Stamm, M. (2009). Begabte Minoritäten. Wiesbaden: VS Verlag für Sozialwissenschaften.

Stamm, M., Niederhauser, M. \& Müller, R. (2009). Begabung und Leistungsexzellenz in der Berufsbildung. Eine empirische Studie zu den Ausbildungsverläufen besonders befähigter Jugendlicher im Schweizer Berufsbildungssystem. Schlussbericht zuhanden der Berufsbildungsforschung des BBT. Universität Fribourg, Departement für Erziehungswissenschaften.

Stanovich, K. E. (1986). Matthew effects in reading: Some consequences of individual differences in the acquisition of literacy. Reading Research Quarterly, 21 (4), 360-407.

Sylva, K., Melhuish, E. C., Sammons, P., Siraj-Blatchford, I., \& Taggart, B. (2008). Effective pre-school and primary education 3-11 project. Final report from the primary phase: Pre- 
school, school and family influences on children's development during key stage 2 [Research Report No. DCSF-RR061]. University of London, Institute of Education.

Treiber, B. \& Weinert, F. E. (1985). Gute Schulleistungen für alle? Psychologische Studien zu einer pädagogischen. Hoffnung. Münster: Aschendorff.

Von Hentig, H. (1985). Die Menschen stärken, die Sachen klären. Ein Plädoyer für die. Wiederherstellung der Aufklärung. Stuttgart: Philipp Reclam.

Voss, J. F. \& Silfies, L. N. (1996). Learning from history text: The integration of knowledge and comprehension skill with text structure. Cognition and Instruction, 14 (1), 45-68.

Walberg, H. J. \& Tsai, S. (1983). Matthew effects in education. American Educational Research Journal, 20 (3), 359-373.

Weinert, F. E. (1997). Psychologie des Unterrichts und der Schule. In: Enzyklopädie der Psychologie, Serie Pädagogische Psychologie, Band 3. Göttingen: Hogrefe.

Weinert, F. E. (2000). Begabung und Lernen: Zur Entwicklung geistiger Leistungsunterschiede. In H. Wagner (Hrsg.), Begabung und Leistung in der Schule. Modelle der Begabtenförderung in Theorie und Praxis (S. 7-24). Bad Honnef: Bock.

Weinert, F. E. \& Helmke, A. (1987). Schulleistungen - Leistungen der Schule oder der. Kinder? Bild der Wissenschaft. 24, 62-73.

Weinert, F. E. \& Helmke, A. (1997). Entwicklung im Grundschulalter. Weinheim: Beltz.

Willms, J. D. (2006). Learning divides: Ten policy questions about the performance and equity of schools and schooling systems. Report prepared for UNESCO Institute for Statistics. Quebec: UNESCO.

Wolter, S. C. \& Coradi Vellacott, M. (2003). Sibling rivalry for parental resources: A problem for equity in education? A six-country comparison with PISA data. Swiss Journal of Socio$\log y, 29$ (3), 377-398.

Zimmer, K., Brunner, M., Lüdtke, O., Prenzel, M. \& Baumert, J. (2007). Die PISA-Spitzengruppe in Deutschland: Eine Charakterisierung hochkompetenter Jugendlicher. In K. A. Heller \& A. Ziegler (Hrsg.), Begabt sein in Deutschland (S. 193-208). Münster: Lit.

Schlagworte: Matthäuseffekte, Leistungsunterschiede, Chancengerechtigkeit, soziale Benachteiligung.

\section{Le riche toujours plus riche et le pauvre toujours plus pauvre? La problématique des effets Matthieu dans des programmes de soutien pédagogique}

\section{Résumé}

Les programmes de soutien pédagogique ont souvent pour effet inattendu de ne pas réduire les différences entre des élèves performants et d'autres moins performants mais plutôt de les augmenter («effets Matthieu»). Cet article examine tout d'abord les preuves empiriques de l'existence d'effets Matthieu en considérant la variabilité des gains d'apprentissage en fonction des types d'écoles fréquentés. Sur la base des résultats de deux enquêtes évaluatives, il présente ensuite un modèle taxonomique des différents bénéfices produits par des programmes de soutien; ce modèle considère à la fois les effets individuels liés aux performances et 
les effets structuraux relatifs à l'accès à ces programmes. Des questions de différences d'ordre psychologique et de politique socio-économique sont ensuite discutées en examinant comment l'effet Matthieu pourrait être en cause lorsque les mesures compensatoires ne produisent pas les effets attendus. La dernière partie de l'article présente des propositions pour l'enseignement pouvant contribuer à la neutralisation des effets Matthieu.

Mots clés: Effets Matthieu, différences de performances, égalité des chances, discrimination sociale.

\section{Ai ricchi sarà dato e ai poveri sarà tolto? La problematica degli effetti Matthew nei programmi di sostegno}

\section{Riassunto}

I programmi di sostegno hanno spesso il sorprendente effetto di non ridurre le differenze tra chi ha risultati buoni e chi ha risultati meno buoni, ma, al contrario, e diversamente da quanto desiderato, di aumentarle ("effetti Matteo). Questo articolo discute in primo luogo le prove empiriche degli effetti Matteo considerando in particolar modo i diversi tipi di apprendimento a dipendenza della scuola frequentata. In secondo luogo, sulla base dei risultati di due studi valutativi, l'articolo presenta un modello di tassonomia per gli apprendimenti non proporzionali nei programmi di sostegno, legato a effetti individuali basati sui risultati e a effetti strutturali nell'accesso. Vengono dunque discusse diverse domande di psicologia differenziale e di politica sociale ed economia relative come approcciare e gestire gli effetti Matteo, ogni volta che delle misure di compensazione non raggiungono i risultati attesi. Infine, vengono formulate alcune conclusioni educative, con il proposito di condurre a maggior equità nei programmi di sostegno.

Parole chiave: Effetti Matteo, differenze di risultati, pari opportunità, discriminazione sociale. 


\title{
The rich get richer and the poor get poorer? Matthew effects and achievement differences in support programmes
}

\begin{abstract}
Support programmes frequently have the surprising effect that they do not, as intended, reduce differences between high-achieving and low-achieving students, but rather increase them ("Matthew effects"). This article first of all discusses empirical documentary evidence of Matthew effects under special consideration of differing learning gains depending on the type of school attended. Thereafter, on the basis of findings from two evaluation studies, it presents a taxonomy model for disproportionate gains in support programmes, which is underpinned by achievement-based individual effects and access-based structural effects. Following this, several differential psychological and social policy/economic questions will be discussed regarding how Matthew effects should fundamentally be dealt with when compensatory measures do not achieve the intended effects. Finally, some educational consequences will be formulated, which might contribute to their equalization.
\end{abstract}

Key words: Matthew effects, achievement differences, equal opportunities, social discrimination. 